\title{
Dai personaggi iconici ai discorsi iconici Strategie e trappole di costruzione dell'immaginario collettivo sul femminismo
}

\section{Federica Turco}

Università degli Studi di Torino

CIRSDe, Centro Interdisciplinare di Ricerche e Studi delle donne e di Genere

federica.turco@unito.it

\begin{abstract}
There are many famous women that, in the last years, has taken the floor, speaking about women (i.e., with the aim to speak about equality and gender violence). Their speeches, in great international visibility contexts, have created a kind of short circuit among their iconicity as celebrities, the iconicity of the context itself and the content of the message.

With a gender point of view and taking into account some famous cases, in the next pages, I'll try to reason about functioning strategies of these texts and about the traps that convert them, in stereotypical and "uniforming" (or at least "not alternative") discourses.
\end{abstract}

\section{Keywords}

Iconicity; Virality; Feminism; Gender; Imaginary

\section{Sommario/Content}

1. If not now, when?

2. Viralità, iconicità e dintorni

3. Iconicità come macchina pigra

4. Concludendo

5. Bibliografia 


\section{Ocula}

Vol 21, No 22 (April 2020) • DOI: 10.12977/ocula2020-6

Federica Turco | Dai personaggi iconici ai discorsi iconici

\section{If not now, when?}

«If not now, when?». Così, il 20 settembre 2014, Emma Watson, attrice britannica anche nota per essere ambasciatrice di UNWomen, ${ }^{1}$ chiudeva un "iconico" discorso tenuto in occasione del lancio della campagna HeForShe, ${ }^{2}$ presso l'ufficio delle Nazioni Unite a New York.

La mobilitazione delle donne famose, e che proprio per godere di una qualche forma di notorietà potevano "prendere la parola" in contesti di grande richiamo mediatico, non era, nel 2014, qualcosa di nuovo o sconosciuto. ${ }^{3}$ Eppure, quel discorso, per una serie di dinamiche e circostanze principalmente co-testuali o contestuali, è certamente considerabile come una sorta di "punto di svolta" 4 nella narrazione sulle donne e sull'empowerment femminile della nostra contemporaneità.

Tra le cause di questa improvvisa attenzione sulla "questione femminile", va sicuramente assegnato un posto importante all'intertestualità che, necessariamente, si crea tra Emma Watson e il personaggio con cui l'attrice è diventata nota al largo pubblico e cioè quello di Hermione Granger, nella serie cinematografica di Harry Potter. Emma Watson è dunque una giovane donna, anagraficamente, la cui giovinezza è ancor più accentuata dall'aver interpretato, al cinema, il ruolo di una bambina. E in quanto giovane donna ha dei sogni e immagina il futuro: come il suo alter ego cinematografico crede nel fatto che gli obiettivi si possano conquistare con la determinazione e la passione, perché solo "credendoci" le cose poi succedono, come in una magia.

$\mathrm{Al}$ di là, però, di questi aspetti "immateriali”, ci sono almeno due questioni, di tipo più sostanziale, che certamente vanno prese in considerazione in questo contesto, la prima di tipo sintattico e la seconda di tipo semantico.

La prima considerazione da fare, che verrà approfondita nei paragrafi a seguire, ha a che fare con i meccanismi della viralità che l'attuale sistema mediatico favorisce. Emma Watson è giovane, dicevamo, e si confronta con un sistema di diffusione dei contenuti della comunicazione che dieci anni fa donne.

1 Entità delle Nazioni Unite per l'uguaglianza di genere e l'empowerment delle

2 Campagna di solidarietà a favore dell'uguaglianza creata da UNWomen. L'obiettivo è principalmente, come dice il suo stesso nome, quello di sollecitare e coinvolgere l'universo maschile nella battaglia per l'uguaglianza di genere e la lotta alle discriminazioni verso le donne.

3 Si possono ricordare, come esempi, i discorsi pubblici tenuti da Emma Thompson nel 1996, da Halle Berry nel 2002 e da Sandra Bullock nel 2010, su temi analoghi.

4 Il riferimento è, qui, principalmente alle teorie sulla sceneggiatura cinematografica e ai concetti di inciting incident, mid point, turning point, climax. Come ci spiegano i manuali di sceneggiatura filmica, se consideriamo le narrazioni come degli archi di trasformazione volti a modificare o a ripristinare delle situazioni di disequilibrio per i soggetti, ad un certo punto della nostra storia avremo sempre un momento "scatenante", in cui vengono svelati i valori di base e gli obiettivi diventano pertinenti rispetto ai percorsi narrativi. Si tratta di quel momento in cui l'attenzione finalmente si focalizza sul dilemma interiore del protagonista della storia e che quindi dà l'avvio al cambiamento vero $\mathrm{e}$ proprio (si vedano per esempio: McKee 1997; Marks 2007; Bandirali, Terrone 2009). 


\section{Ocula}

Vol 21, No 22 (April 2020) • DOI: 10.12977/ocula2020-6

Federica Turco | Dai personaggi iconici ai discorsi iconici

non esisteva, quello dei social network. Più o meno coscientemente (il che è solo accidentale e non particolarmente rilevante ai fini di questo saggio), il suo discorso alle Nazioni Unite è pensato per un pubblico, quello di Internet, che non solo ha dimensioni considerevoli in termini numerici, ma che è solito mettere in scena un ben preciso comportamento di fronte ai contenuti della comunicazione e alla loro "ripetibilità" sistematica.

Come detto, di questo parlerò ampiamente più avanti.

Vorrei qui, invece, soffermarmi sulla seconda questione, che ho detto essere di tipo "semantico", perché ha a che fare, più nello specifico, con gli effetti di senso che il discorso di Emma Watson in primis, ma poi anche una serie di altre orazioni pubbliche che sono seguite a questa, hanno avuto.

\section{Di seguito i passaggi salienti del discorso:}

Today we are launching a campaign called "HeForShe." I am reaching out to you because I need your help. We want to end gender inequality-and to do that we need everyone to be involved. This is the first campaign of its kind at the UN: we want to try and galvanize as many men and boys as possible to be advocates for gender equality. And we don't just want to talk about it, but make sure it is tangible. I was appointed six months ago and the more I have spoken about feminism the more I have realized that fighting for women's rights has too often become synonymous with man-hating. If there is one thing I know for certain, it is that this has to stop. For the record, feminism by definition is: «The belief that men and women should have equal rights and opportunities. It is the theory of the political, economic and social equality of the sexes». [...]

When at 14 I started being sexualized by certain elements of the press. When at 15 my girlfriends started dropping out of their sports teams because they didn't want to appear "muscly". When at 18 my male friends were unable to express their feelings. I decided I was a feminist and this seemed uncomplicated to me. But my recent research has shown me that feminism has become an unpopular word. Apparently, I am among the ranks of women whose expressions are seen as too strong, too aggressive, isolating, anti-men and, unattractive. Why is the word such an uncomfortable one? [...]

Men-I would like to take this opportunity to extend your formal invitation. Gender equality is your issue too. [...]

If we stop defining each other by what we are not and start defining ourselves by what we are-we can all be freer and this is what HeForShe is about. It's about freedom. I want men to take up this mantle. So their daughters, sisters and mothers can be free from prejudice but also so that their sons have permission to be vulnerable and human too-reclaim those parts of themselves they abandoned and in doing so be a more true and complete version of themselves. You might be thinking who is this Harry Potter girl? And what is she doing up on stage at the UN. It's a good question and trust me, I have been asking myself the same thing. I don't know if I am qualified to be here. All I know is that I care about this problem. And I want to make it better. [...]

Because the reality is that if we do nothing it will take 75 years, or for me to be nearly a hundred before women can expect to be paid the same as men for the same work. 15.5 million girls will be married in the next 16 years as children. And at current rates it won't be until 2086 before all rural African girls will be able to receive a secondary 


\section{Ocula}

Vol 21, No 22 (April 2020) • DOI: 10.12977/ocula2020-6

Federica Turco | Dai personaggi iconici ai discorsi iconici

education. If you believe in equality, you might be one of those inadvertent feminists I spoke of earlier. And for this I applaud you. We are struggling for a uniting word but the good news is we have a uniting movement. It is called HeForShe. I am inviting you to step forward, to be seen to speak up, to be the "he" for "she". And to ask yourself if not me, who? If not now, when? (Emma Watson, 20 settembre 2014, ONU). ${ }^{5}$

Come si può facilmente notare scorrendo le parole dell'attrice, dopo alcune premesse di tipo definitorio sul femminismo e dopo la "chiamata all'azione" verso la popolazione maschile (necessaria visti gli obiettivi stessi della campagna HeForShe), l'attenzione si concentra essenzialmente su un tema molto specifico, che è quello del tempo. Questo topic ${ }^{6}$ viene introdotto attraverso la scansione puntuale delle fasi della vita dell'attrice (il che, è ovvio, serve anche a meglio posizionare il suo diritto di parola sull'argomento, proprio a partire dall'essere stata "la maghetta di Harry Potter", eliminando così l'eventuale dissonanza cognitiva che potrebbe nascere dall'accostare una "bambina", cinematograficamente parlando, alle lotte femministe) e trova il suo climax proprio nel motto conclusivo: «Se non ora, quando?».

A quanto pare, dunque, il "tempo", oltre ad essere il topic di questo discorso, è anche il suo focus: abbiamo bisogno di parlare di tempo, perché la questione, il problema è appunto il tempo, sia in termini assoluti, filosofici potremmo dire, sia in termini pratici.

I pregiudizi di genere, ci dice Emma/Hermione, esistono da sempre e accompagnano la vita delle donne, fin dalla loro nascita. Per questo riguardano tutti, uomini e donne: entrano nella nostra vita insieme a tutti i livelli di relazione che intratteniamo con le persone che ne fanno parte (madri, figlie, amiche, compagne, mogli...). A questa idea del tempo di tipo trasversale ("il tempo della vita") si sovrappone un'idea di tempo di tipo contingente ("il tempo dell'azione"). Il suggerimento è quello di un tempo relativo e soggettivo, kantiano in qualche modo, che ci costringe a prendere coscienza del nostro bisogno di governarne lo scorrere.

Interessante è soprattutto, come anticipato, il motto finale, una specie di atto linguistico perlocutorio che si pone l'obiettivo di far muovere, metaforicamente, le persone in una direzione diversa.

Molte cose si possono dire intorno alla scelta del motto «Se non ora, quando?», che ha una lunga storia di citazioni, più o meno corrette e più o meno consapevoli. Sappiamo che la genesi dell'espressione si deve al rabbino Hillel il Vecchio, che la usò nel 60 a.C. circa e che compare nel Pirké Avoth, le Massime dei Padri, la parte più antica del Talmud. ${ }^{7}$

5 È possibile ascoltare una versione integrale del discorso al link che segue (data dell'ultima consultazione: 21 maggio 2019): <https://www.youtube.com/watch?v=gkjW9PZBRfk>.

6 Intendiamo il concetto di "topic" come ciò di cui si parla, ovvero il tema, l'argomento, in contrapposizione al focus di un testo che è invece ciò che viene, appunto, affermato, su tale tema (su questo punto si veda Ferraro, Santangelo 2013).

7 «Se io non sono per me, chi è per me? E se io sono solo per me stesso, cosa sono? E se non ora, quando?» (Pirké Avoth: 1, 14). 


\section{Ocula}

Vol 21, No 22 (April 2020) • DOI: 10.12977/ocula2020-6

Federica Turco | Dai personaggi iconici ai discorsi iconici

Esula senza dubbio dagli obiettivi di questo contributo (nonché dalle competenze di chi scrive) una disamina del senso di questo motto nel suo contesto di origine, ma senza dubbio è innegabile l'approccio di tipo etico che ha la frase: fu pronunciata per favorire una riflessione sul bene e sul male, sul giusto e sullo sbagliato, in tema di relazione col prossimo ( $«$ se io non sono per me, chi è per me?»), di identità («e se io sono solo per me stesso, cosa sono?») e di motivazione all'agire umano («e se non ora, quando?»).

Tre questioni, queste, che facilmente rintracciamo anche nella storia successiva del motto (anche nella sua versione più corta): nell'omonimo romanzo di Primo Levi del 1982, per esempio, dedicato alla storia di un gruppo di partigiani ebrei, incontriamo il topic dell'avventura come chiave per una vita piena, fatta di amori e tradimenti, di sbagli e correzioni, di esaltazioni e delusioni, che non sarebbero possibili senza una certa dose di rischio e di predisposizione all'azione. Senza riserve e senza titubanze. È un romanzo, quello di Levi, dell'hic et nunc.

È questo il senso, anche, del nome che si è dato il movimento italiano di donne nato nel 2011, che si propone di lavorare sulla rappresentanza e sulla rappresentazione delle donne, sulla violenza di genere e sul welfare, con l'obiettivo di "non perdere tempo" nella lunga strada che bisogna compiere per raggiungere una reale e sostanziale parità tra uomini e donne.

Anche nei suoi usi più populisti (vi è infatti una lunga storia di attribuzioni, talvolta anche errate, nel discorso politico soprattutto americano ${ }^{8}$ ), la frase ha comunque l'intento di proporre una relazione, se non di ostilità quanto meno di conflitto con il tempo.

Il conflitto con "il tempo che scorre inesorabile" è dunque la chiave di lettura del discorso di Emma Watson; un tempo, come dicevamo, necessariamente soggettivo, ma che, nel parametro dell'urgenza, innesca un movimento che cessa di essere del singolo individuo e diventa collettivo.

Impostazione che, peraltro, troviamo ancora in diverse occasioni successive: Patricia Arquette, premiata durante la Notte degli Oscar 2015 come miglior attrice non protagonista, declama dal palco «It's our time to have rights» (corsivo mio); Oprah Winfrey, ai Golden Globes 2018, ${ }^{9}$ riceve un premio per la sua carriera e nel suo discorso di ringraziamento parla proprio della necessità, per le donne, di aprire una nuova fase temporale: «And for too long, women have not been heard or believed if they dared to speak their truth to the power of those men. But their time is up. Their time is up. Their time is up» (corsivo mio); sempre nel 2018, Viola Davis pronuncia un lungo discorso dal palco della Women's March di New York ripetendo più volte la parola «today», mentre Frances McDormand, premio Oscar come miglior attrice protagoni-

8 Da J.F. Kennedy a R. Regan, da G. Romney a I. Trump, ecc.

9 Edizione che è stata segnata dall'allora recente "scandalo Weinstein" e in cui di fatto nasce il movimento Time's Up!, che accompagna e incornicia il motto \#meetoo e che coinvolge un altissimo numero di star holliwoodiane, tra cui Maryl Streep. Per approfondimenti: <https://www.ilpost.it/2018/01/01/times-up-donne-molestie-sessuali>; <http://time.com/5189945/whats-the-difference-between-the-metoo-and-times-up-movements $>$. 


\section{Ocula}

Vol 21, No 22 (April 2020) • DOI: 10.12977/ocula2020-6

Federica Turco | Dai personaggi iconici ai discorsi iconici

sta, ammonisce le sue colleghe della platea a «muoversi» (letteralmente) per raccontare la propria storia di donne, per presentare e portare avanti i propri progetti «senza perdere tempo»; nel 2019 di nuovo durante i Golden Globes ritroviamo lo stesso monito, questa volta portato avanti da Glenn Close, che racconta la storia della propria madre, la quale in tardissima età si rimproverava d'aver realizzato troppo poco e di non avere più ormai il tempo per farlo (con l'obiettivo, quindi, di dire alle altre donne di non aspettare che sia troppo tardi e di muoversi subito).

Tutti questi discorsi hanno alcune cose in comune: (i) sono tutti pronunciati da donne note, donne che possiamo considerare in qualche modo delle icone, per la loro notorietà, per la notorietà dei personaggi che interpretano al cinema, per i ruoli che rivestono nella società civile e nei media; (ii) sono tutti incentrati su una strategia che, attraverso il tema del tempo, mette in scena il codice passionale dell'ansia, dell'urgenza, dell'affanno; (iii) sono tutti diventati rapidamente virali.

È proprio questa viralità, che costituisce, a mio avviso, la base della loro iconicità e, al tempo stesso, lo svuotamento di senso che, nella loro diffusione, finiscono per subire.

\section{Viralità, iconicità e dintorni}

Che cosa significa "viralità"? Fornire una spiegazione che ambisca ad avere valore definitorio di un fenomeno che, oltre ad essere in divenire continuo, è anche oggetto di una visibilità prima sconosciuta, non è ovviamente cosa semplice. Questo perché, in primis, il rischio è quello di cadere nella trappola del luogo comune e di non riuscirne a considerare gli aspetti astratti, rifacendosi soltanto agli aspetti pragmatici (ovvero: quali sono gli effetti della viralità, piuttosto che quali sono le cause della sua origine).

Secondo il vocabolario della lingua italiana, tale termine corrisponde alla capacità di informazioni e messaggi di propagarsi a un grande numero di destinatari, trasmettendosi dall'uno all'altro, anche in virtù delle caratteristiche degli strumenti di comunicazione contemporanei, similmente ad una malattia contagiosa (e infatti la radice etimologica è appunto virus).

Noto è, proprio per questo motivo, l'uso della metafora del contagio per descrivere tale fenomeno: all'interno di un certo contesto culturale le idee nascono e si propagano tra le popolazioni "per contagio" e si stabilizzano in codici, forme e temi, creando di conseguenza le rappresentazioni culturali. ${ }^{10}$ Si intercetta, in questo tipo di definizione, una certa passività del ricevente, che come in una malattia contagiosa, appunto, si limita ad essere esposto ad un elemento contaminante, suo malgrado, senza mai intervenire direttamente (con un atto di volontarietà) nel processo. Volli (2016) suggerisce una ulteriore declinazione della diffusione della cultura, attraverso il "modello dell'untore":

10 I riferimenti sono numerosi. Si possono vedere, per esempio: Marchetti (1991a) e (1991b); Sperber (2000); Manetti, Barcellona, Rampoldi (2003) (e in particolare il contributo di Eric Landowski). 


\section{Ocula}

Vol 21, No 22 (April 2020) • DOI: 10.12977/ocula2020-6

Federica Turco | Dai personaggi iconici ai discorsi iconici

Dal punto di vista epidemiologico, il modello del contagio di spiegazione delle epidemie (che naturalmente è quello giusto, di cui non solo da secoli conosciamo i meccanismi ma oggi abbiamo conoscenza precisa del funzionamento microbiologico) si contrappone non solo al modo di diffusione di malattie non epidemiche come i tumori o gli ictus, ma anche al modello dell'untore che spiega l'estendersi di un morbo con l'azione volontaria. [...] "Modello dell'untore" e "modello del contagio" sono incompatibili, alternativi sul piano terapeutico ed epidemiologico, oltre che su quello morale - dato che il modello dell'untore è stato spesso usato insieme alla teoria del complotto per eliminare nemici o perseguitare minoranze.

$[\ldots]$

Dunque, nel momento in cui si dica che i contenuti culturali sono virali, si afferma che essi sono indifferenti all'azione dei soggetti che ne sono portatori, di quelli che li trasmettono come di quelli che li ricevono. Questi sono solo veicoli, contenitori, alimenti, ma i contenuti, se sono virali, vanno per conto loro. Dunque il viralismo implica una forma di antiumanismo, di depotenziamento della responsabilità individuale almeno nella formazione dei contenuti culturali (Volli 2016: 61-62).

Una prima questione teorica di rilievo, dunque, secondo Volli, è il problema dell'agentività: quando si parla di viralità, chi è l'agente? Chi è il soggetto che è responsabile di immettere nel discorso, quel tipo di contenuto? E soprattutto, c'è un responsabile?

La metafora del contagio va accettata, sì, ma solo in maniera, per così dire, "calmierata": la produzione dei contenuti della comunicazione è sempre azione di qualcuno che ne è responsabile, sia quando tali contenuti vengono accettati e rimessi in circolo tout court, sia quando vengono modificati e rimaneggiati (Turco 2016b). In particolare nella circolazione dei contenuti on line, il debrayage ${ }^{11}$ è prova dell'esistenza stessa dell'azione enunciativa. Esiste una sorta di pragmatica della comunicazione virale, grazie alla quale (o a causa della quale), i testi fungono come amplificatori dei processi di agency, che sussistono non solo a monte ma anche a valle del percorso di comunicazione. ${ }^{12}$

Oltre alla questione del "chi", c'è poi la questione del "che cosa". Come si costruisce un discorso che diventi virale? Qual è il suo contenuto? Quanto esso è rilevante nell'economia della circolazione delle idee e della costruzione dei modelli culturali che da esse scaturiscono?

Certamente uno dei lavori più significativi nello studio del fenomeno della viralità, lo dobbiamo a Henry Jenkins, secondo cui la spreadability è l'insieme delle risorse e delle capacità tecniche che permettono la maggiore diffusio-

11 Nella teoria dell'enunciazione, il débrayage è l'operazione con cui l'istanza dell'enunciazione disgiunge e proietta fuori di sé, al momento dell'atto di linguaggio e in vista della manifestazione, certi termini legati alla sua struttura di base per costituire così gli elementi fondatori dell'enunciato-discorso (Greimas e Courtés 1979). Letteralmente vuol dire "disinnesco": è la separazione del testo enunciato dalla situazione dell'enunciazione vera e propria.

12 A questo proposito, Marino definiva i testi virali come dei testi ergodici, e cioè faticosi, perché per essere pienamente sensati per l'utente, richiedono uno sforzo (Marino 2015). 


\section{Ocula}

Vol 21, No 22 (April 2020) • DOI: 10.12977/ocula2020-6

Federica Turco | Dai personaggi iconici ai discorsi iconici

ne di taluni contenuti (incluse le strutture economiche), delle caratteristiche del sistema mediatico che ne permette la capillare replicabilità (senza i social network, probabilmente, non avremmo contenuti virali, ndr), dei requisiti del testo stesso, che devono essere in qualche misura rimodellabili in modo originale (Jenkins, Ford, Green 2013). Il testo, come suggeriscono Dusi e Spaziante (2006) non è più qualcosa di fisso e finito, ma diventa "plurale", nel senso di acquisire il suo significato (e la sua forza in termini di capacità di incidere nella storia delle idee) solo come frutto di successive sovrapposizioni e dunque in maniera diacronica più che sincronica.

C'è una sorta di logica del "frammento": il testo potenzialmente virale è scomponibile in blocchi, in frammenti, citazioni, momenti singoli, slogan che, selezionati dal singolo individuo in modo che quei singoli passaggi confermino in qualche modo le sue preesistenti convinzioni ideologiche, diventano poi parte integrante della cultura. Mi sembra che si possa richiamare qui la nota definizione echiana di "sgangherabilità" (1977 e 2000): quella proprietà di un'opera, dal profondo contenuto emozionale, di essere smembrata e goduta un pezzo alla volta. Condizione, questa, per essere non solo un'opera di successo, ma addirittura di culto. Ma questi pezzi "sgangherabili" non sono casuali, devono trovare collocazione nelle radici più arcaiche dell'inconscio, essere in qualche modo degli archetipi, fare leva su convinzioni che siano apparentemente individuali e invece profondamente collettive (devono avere, potremmo riassumere semplificando, una qualche forma di destinazione esterna al soggetto, essendo "sociali", più che "individuali").

Tali frammenti agiscono nel testo e nelle sue derive interpretative come punctum (Barthes 1980), colpiscono emotivamente il lettore, grazie ad un passaggio simbolico dal piano della persuasione (il discorso che Emma Watson fa nel 2014 non si limita ad un "far fare"), al piano del sentire (perché il suo obiettivo è "far essere" parte di una collettività a cui è affidata un'urgenza: il tempo che passa).

E infatti proprio così, come frammento, è stato preso e rielaborato, manipolato e riadattato da innumerevoli discorsi successivi, come si è visto, finendo per dare il nome a un vero e proprio movimento (Time's Up).

Sembra dunque che ciò che spiega la trasformazione dei discorsi pubblici in discorsi iconici, sia la loro capacità di essere virali. Capacità che è data dalla loro stessa configurazione frammentaria, fatta di giustapposizioni di grandi questioni emotive, più che di una reale struttura di senso.

\section{Iconicità come macchina pigra}

Perché l'iconicità di tali discorsi regga, però, deve sussistere una sorta di bilanciamento tra la norma e lo scarto dalla norma stessa. I discorsi di queste donne famose, che si ergono a paladine dei diritti delle donne, funzionano perché mettono in scena, da un lato, aspettative già consolidate e diffuse, che siano appunto presenti come matrici di grandi valori collettivi, «la cui conoscenza e memorizzazione da parte di tutti [...] è funzionale alla trasmissione di una certa cultura [...] e della sua specifica visione del mondo» (Santangelo 


\section{Ocula}

Vol 21, No 22 (April 2020) • DOI: 10.12977/ocula2020-6

Federica Turco | Dai personaggi iconici ai discorsi iconici

2016: 283). Dall'altro lato essi, però, si manifestano come significativi rispetto al mainstream e acquisiscono un certo regime di visibilità, proprio in ragione di questo scostamento. L'iconicità è insomma una macchina pigra, nel senso echiano: lascia spazi bianchi che vanno riempiti (Eco 1979). Perché questo avvenga gli elementi di novità non possono prevalere su quelli di ripetibilità, ma devono con essi scendere a patti.

La garanzia di questo risultato è data, a mio avviso, da elementi sintattici e non da elementi semantici, è data cioè da quella strategia di frammentazione di cui parlavamo poco sopra e che funziona attraverso lo svuotamento di senso. Il motivo per cui i discorsi di cui sopra (da Emma Watson in poi) incarnano così bene l'idea di iconicità che abbiamo ${ }^{13}$ è che essi non creano nuovi contenuti, semmai si posizionano in un regime di contraddizione con i discorsi già diffusi. È il passaggio da $\mathrm{A}$ a non $\mathrm{A}^{14}$ del quadrato semiotico (rappresentazione grafica dei valori che sono alla base di una narrazione e delle trasformazioni che li riguardano), un passaggio, dunque, che non implica una riqualificazione della posizione (che sarebbe, eventualmente, più semanticamente rilevante), ma semplicemente un movimento di differenziazione. Differenziazione che, peraltro, vale indipendentemente dalla direzione. Leone parla di «ipertrofia del livello dell'ideologia sintattica a detrimento di quello dell'ideologia semantica» (Leone 2016: 205).

Il "tempo", trend topic dei nostri iconici discorsi, diventa proprio una variabile di questo sistema delle differenze: "non c'è più tempo" corrisponde a "domani deve essere diverso da ieri". Che a questo corrisponda un chiaro posizionamento su che cosa significhi il passato da cui prendere le distanze, che caratteristiche ha, come si configura nella storia delle donne e del femminismo, non è dato di sapere. $\mathrm{E}$ ancor meno è valorizzato in maniera costruttiva il fare del domani. Casomai siamo di fronte ad una "storia del dire".

La conseguenza principale di questo svuotamento semantico è che, nel professare una posizione differente, appunto, i discorsi a cui si riferisce questo saggio finiscono per essere estremamente stereotipici e per aderire a quel mainstream da cui si dichiarano distanti, istituendo un mondo binario fatto di maschi predatori e colpevoli e donne vittime e innocenti, universo questo da cui ormai i gender studies propriamente detti hanno, da tempo, preso le distanze.

\section{Concludendo}

Mi avvio a parziali e provvisorie conclusioni, ponendomi ancora qualche domanda: quale relazione sussiste tra iconicità e culto? $\mathrm{E}$ in che modo questi due elementi sono connessi con i modelli culturali e gli immaginari collettivi?

13 Non va dimenticato che "icona" è, in greco, "immagine": l'icona è un segno che stabilisce un rapporto di somiglianza tra significante e significato, nel linguaggio comune viene considerato iconico ciò che è percepito come conforme all'immagine del simboleggiato, del raffigurato, del rappresentato.

14 Su questo si veda un ragionamento analogo che Massimo Leone conduce a proposito della formazione dell'opinione pubblica in Rete (Leone 2016). 


\section{Ocula}

Vol 21, No 22 (April 2020) • DOI: 10.12977/ocula2020-6

Federica Turco | Dai personaggi iconici ai discorsi iconici

Molte cose già sono state dette: si è parlato di ripetizione e ripetibilità, di bilanciamento tra norma e scarto, di frammento e sgangherabilità, di selezione e ordine. Si è anche parlato della relazione tra gli elementi testuali e gli elementi contestuali nella costruzione dei percorsi di riconoscibilità dei testi.

Se ragioniamo sulla parola "culto", ci accorgiamo immediatamente di uno slittamento importante tra il suo significato originale, di pratica religiosa e quello, invece, contemporaneo più profano e secolarizzato. Eppure, sta proprio nell'associazione tra questi due livelli la risposta alle nostre domande: il culto come pratica religiosa motiva la sua forza di coinvolgimento (che era già virale, in qualche modo, in momenti storici che precedevano l'idea stessa di viralità nel modo in cui la intendiamo oggi) nella struttura sintattica del rito: un insieme fisso e ordinato di elementi che si susseguono a prescindere dal loro valore in termini di significato e che semmai mettono in scena proprio la variabile temporale (nei riti c'è un prima, in cui il soggetto è in una determinata posizione, e un dopo, in cui il soggetto risulta trasformato - ad esempio sposato, incoronato, eletto, ecc.). Allo stesso modo, in questi eventi mediatici contemporanei ${ }^{15}$ che sono stati il luogo del nascere dei discorsi iconici riferimento del presente saggio, la ritualità formale rende spettacolare (anche esteticamente) la parola pronunciata da taluni personaggi (che peraltro tanto assomigliano a idoli veri e propri). Come si diceva, che questo abbia poi a che fare realmente con ciò che essi dicono, piuttosto che con il fatto stesso di dire, è altro discorso.

Se noi definiamo i modelli culturali come configurazioni discorsive ricorrenti, capaci di reggere (dare coerenza a) intere enciclopedie di segni (Turco 2016a), possiamo facilmente capire come essi diventino la florida base degli immaginari collettivi; immaginari a cui noi ci rifacciamo e che accettiamo come basi credibili del nostro agire quotidiano. È il loro essere dei frame, degli script a renderli condivisibili.

Le rappresentazioni testuali delle donne non differiscono da questo sistema. Come ha sostenuto Cristina Demaria (2003), anche le rappresentazioni del soggetto sessuato influenzano e sono determinate dall'enciclopedia e dai meccanismi dell'interpretazione; emergono e sono frutto dei discorsi e delle prassi enunciative di una cultura. Nelle rappresentazioni testuali delle donne confluiscono tutte le isotopie, ovvero le ridondanze semantiche che rendono leggibile e interpretabile una narrazione, e le metafore dell'esperienza quotidiana e, viceversa, tali rappresentazioni contribuiscono alla formazione di abiti, ${ }^{16}$ interpretazioni, definizioni dell'identità sessuale. Sono modelli.

Il prototipo conflittuale posto in essere dai modelli testuali di donne che scorgiamo nei discorsi presentati in questo breve scritto, produce degli im-

15 Mi rifaccio qui alla definizione di evento mediale, così come risulta in Katz e Dayan (1993): un genere narrativo che usa il carattere specifico del mezzo (nel nostro caso, direi, soprattutto i social network e solo secondariamente la televisione, dove tali discorsi sono stati, in primis, trasmessi), per dirigere l'attenzione di grandi quantità di persone verso lo stesso contenuto.

16 Dove per "abito" intendiamo il processo continuo di semiosi in cui confluiscono aspettative, percezioni, pratiche, ecc. Tali abiti sono sia il risultato sia la condizione della produzione sociale del significato. 


\section{Ocula ${ }^{22}$}

Vol 21, No 22 (April 2020) • DOI: 10.12977/ocula2020-6

Federica Turco | Dai personaggi iconici ai discorsi iconici

maginari esclusivi, più che inclusivi, dove il processo di normalizzazione (del conflitto stesso) risulta prevalere su quello, auspicato dal femminismo attuale, di posizionamento nella differenza.

Ci ritroviamo di fronte a soggetti molto poco "eccentrici" (de Lauretis 1999), la cui identità viene confermata proprio da quel processo di replicabilità, di viralità, che come detto attinge ad archetipi e modelli sedimentati e tradizionali.

\section{Bibliografia}

Bandirali, Luca; Terrone, Enrico

2009 Il sistema sceneggiatura. Scrivere e descrivere i film, Torino, Lindau.

Barthes, Roland

1980 La Chambre claire. Note sur la photographie, Parigi, Gallimard.

Dawkins, Richard

1976 The Selfish Gene, Oxford, Oxford University Press.

Dayan, Daniel; Katz, Elihu

1993 Le grandi cerimonie dei media, la storia in diretta, Bologna, Baskerville.

de Lauretis, Teresa

1999 Soggetti eccentrici, Milano, Feltrinelli.

Demaria, Cristina

2003 Teorie di genere. Femminismo, critica postcoloniale e semiotica, Milano, Bompiani.

Dusi Nicola; Spaziante, Lucio

2006 Remix-remake: Pratiche di replicabilità, Roma, Meltemi.

Eco, Umberto

1977 “Casablanca, o la rinascita degli dei”, in Dalla periferia dell'impero, Milano, Bompiani, pp. 138-143.

Eco, Umberto

1979 Lector in fabula, Milano, Bompiani.

Eco, Umberto

2000 "Libri Sgangherati”, in La bustina di Minerva, Milano, Bompiani, pp. 239240.

Edelman, Gerald M.

2007 Seconda natura. Scienza del cervello e conoscenza umana, Milano, Raffaello Cortina Editore.

Ferraro, Guido; Santangelo, Antonio (a cura di)

2013 Uno sguardo più attento. I dispositivi di senso dei testi cinematografici, I Saggi di Lexia, Roma, Aracne. 


\section{Ocula}

Vol 21, No 22 (April 2020) • DOI: 10.12977/ocula2020-6

Federica Turco | Dai personaggi iconici ai discorsi iconici

Greimas, Algirdas J.; Courtés, Joseph

1979 Sémiotique. Dictionnaire raisonné de la théorie du langage, Paris, Hachette.

Jenkins, Henry; Ford, Sam; Green, Joshua

2013 Spreadable Media: Creating Value and Meaning on a Networked Culture, New York and London, New York University Press.

Kant, Immanuel

1781/1787 Kritik der reinen Vernunft, Riga, Verlag Johann Friedrich Hartknoch (ed. it. Critica della ragion pura, Roma-Bari, Laterza, 2000).

Landowski, Eric

2003 "Al di qua o al di là delle strategie: la presenza contagiosa", in Manetti, G., Barcellona, L. e Rampoldi C. (a cura di), Il contagio e i suoi simboli, Pisa, Edizioni ETS.

Leone, Massimo

2016 "Il bastian contrario nella rete. Pattern rituali di formazione dell'opinione nella semiosfera dei social networks", Lexia Nuova Serie, 25-26, pp. 173-210.

Manetti, Giovanni; Barcellona, Luca; Rampoldi Cora (a cura di)

2003 Il contagio e $i$ suoi simboli, Pisa, Edizioni ETS.

Marchetti, Cesare

1991a "A Forecasting Model for Research and Innovation Activities in Selected Areas: A Support for Strategic Choices”, paper presentato all'International Course on Research and Innovation Management, Venezia, ora in $<\mathrm{http}: / / \mathrm{www}$ cesaremarchetti.org/archive/scan/MARCHETTI-085.pdf>.

Marchetti, Cesare

1991b "Modeling Innovation Diffusion", in Henry B. (a cura di), Forecasting Technological Innovation, Kluwer Academic Publishersw, MA, Norwell; ora in <http://cesaremarchetti.org/archive/scan/MARCHETTI-035.pdf $>$.

Marino, Gabriele

2014 "Keep calm and Do the Harlem Shake: meme, Internet meme e meme musicali”, in Pezzini I., Spaziante L. (a cura di), Corpi mediali. Semiotica e contemporaneità, Pisa, Edizioni ETS.

Marino, Gabriele

2015 "Semiotics of Spreadability: A sistematic approach to Internet Memes and Virality", in Paschalidis G. (a cura di), Semiotics of the Web (Punctum monographic issue, 1,1), pp. 43-66.

Marino, Gabriele; Thibault, Mattia (a cura di)

2016 Viralità. Per un'epidemiologia del senso, Lexia Nuova Serie, 25-26, Roma, Aracne.

Marks, Dora

2007 Inside story: the power of the transformational arch, Studio City, Three mountain press.

McKee, Robert

1997 Story. Style, structure, substance and the principles of screenwriting, New York, HarperCollins. 


\section{Ocula}

Vol 21, No 22 (April 2020) • DOI: 10.12977/ocula2020-6

Federica Turco | Dai personaggi iconici ai discorsi iconici

Santangelo, A.

2016 "I regimi di visibilità sui media. Riflessioni sociosemiotiche sui meccanismi della viralità”, Lexia Nuova Serie, 25-26, pp.271-283.

Sperber Dan

2000 "An objection to the memetic approach to culture", in Aunger R. (a cura di), Darwinizing Culture: The Status of Memetics as a Science, Oxford, Oxford University Press.

Turco, Federica

2016a "It's all President's fault. Tricks, conspiracies, corruptions in American TV series set in the White House: Homeland and Scandal", Lexia Nuova Serie, 23-24, pp. 429-444.

Turco, Federica

2016b "L’ideologia gender esiste davvero? Tra omofobia e libri messi al bando, percorsi narrativi di un falso mito", Lexia Nuova Serie, 25-26, pp. 251-268.

Volli, Ugo

2016 "Il contagio della metafora”, Lexia Nuova Serie, 25-26, pp. 55-72.

Federica Turco è dottore di ricerca in Scienze e Progetto della Comunicazione, presso l'Università degli Studi di Torino e insegna Semiotica del gender presso lo stesso Ateneo. Tra le sue ultime pubblicazioni: "It's all the President's Fault. Tricks, Conspiracies, and Cor-ruption in the American TV Series Set at the White House: Homeland and Scandal", in Lexia Nuova Serie, $n^{\circ}$ 23-24, 2016; "L'ideologia gender esiste davvero? tra omofobia e libri messi al bando, percorsi narrativi di un falso mito", in Lexia Nuova Serie, $\mathrm{n}^{\circ}$ 25-26, 2016; "Stupri, femminicidi, botte. Sulle derive catastrofiche del senso del corpo delle donne”, in Idone Cassone V., Surace B., Thibault M. (eds.), I discorsi della fine. Catastrofi, disastri, apocalissi, I saggi di Lexia, 2018. 